\title{
HIGH ACCURACY ANALYSIS OF ARBITRARY MODES IN TAPERED DISK-LOADED STRUCTURES
}

\author{
L.F. WANG ${ }^{*}$, Y. Z. LIN $^{1}$, T. HIGO ${ }^{2}$ and K. TAKATA ${ }^{2}$ \\ ${ }^{1}$ Department of Engineering Physics, Tsinghua University,Beijing 100084, P.R. China \\ ${ }^{2}$ High Energy Accelerator Research Organization (KEK), Tsukuba, Ibaraki 305-0801, Japan
}

\begin{abstract}
In this paper, a high-precision eigenmode-computation analysis of arbitrary azimuthal mode numbers in periodic disc-loaded structure based on variational method will be discussed. It allows for rounding the edge of a disk hole without any approximation in shape treatment and calculates the modes exactly synchronous to the beam. It converges much faster than the mesh-based computer code. Good agreement was observed between the results of variational method and those of other methods.
\end{abstract}

\section{INTRODUCTION}

In the past, various numerical methods were developed for the analysis of the higher order modes in a cell[1-4], such as KN7C[5], TRANSVRS[6], URMEL[7], MAFIA[8], and $\Omega 2[9]$, SUPERFISH[10]. The codes KN7C and TRANSVRS can calculate the longitudinal and transverse modes with any phase advance per period, though imposing two approximations: an infinite periodic structure and a flat beam hole surface parallel to the beam axis instead of round edge. Because of the latter assumption, the calculated wake field is not accurate enough[1]. Though these two codes have been very successfully applied to the actual design of structures, they need to be further improved if they are to be used for cells with a round edge beam hole. This is one of the motives for developing the present code.

On the other hand, such codes as SUPERFISH, URMEL, $\Omega 2$ and MAFIA can represent the structure by filling with a mesh so that these can calculate the modes in cells of any shape. However, our method still has its advantages in many aspects. The variational method can describes the structure geometry without any approximation. Therefore it is a better way in the geometry treatment than various mesh-based methods. The fields in the variational method are given as a series expansion, which derives from the Maxwell equations. The calculated fields converge on the true ones very fast when the number of terms increases. Also, it uses less memory than the mesh-based method. The accuracy depends on the terms number used. The frequency accuracy is around $10^{-7}$ for the acceleration mode of our $\mathrm{X}$-band structure with a number of terms more than 55 . One can get a sufficiently accurate calculation by increasing the number of terms. Our method can be used to calculate both monopole modes and all azimuthally varying modes with any phase shift per cell, which means that it can calculate all kinds of field in the disk-loaded

*Corresponding author,wanglf@post.kek.jp structure (any azimuthal mode, any synchrotron phase, any frequency or passpand). The fields are expressed as simple formula. Therefore it can be conveniently used to serve as input for other purpose calculation, such as the base vectors for equivalent circuit model or open-mode expansion model [11].

The present code is named LONGTRANSVRS.

\section{THEORY}

The theory of variational approach is a well-known method and is basically a mathematical forerunner of the finite element method. In the classical formulation the problem is to find the unknown function or functions that extremize (maximize, minimize) or get stationary under the specified boundary condition. The fields with steadystate sinusoidal time dependence of $e^{j \omega t}$ in vacuum space can be found by making the following value minimum

$$
J=j k \int\left(Z_{0} \mathbf{H}\right)^{*} \times \mathbf{E} \cdot \mathbf{n} d S .
$$

where $k=\omega \sqrt{\varepsilon_{0} \mu_{0}}$ is the propagation constant, $\mathbf{n}$ is the unit vector outward normal to the surface $S$. The variational form eq.(1) has no further limitation on the trial function on the metal boundary, but the non-metal boundary condition should be satisfied by the trial function.

The accelerator structure with which we are concerned is a conventional disk-loaded cylindrical waveguide as shown in Figure 1. The whole acceleration structure is divided into the inner and outer regions, separated at the common boundary at $r=r_{c}$. The inner region is characterized by the fact that it has a rounded edge of the disk hole as part of boundary surface.

The Hertz vectors $\Pi_{h}=\hat{z} \Pi_{h, z}$ and $\Pi_{e}=\hat{z} \Pi_{e, z}$ are chosen to simplify the solution. In the inner traveling wave region, the Hertz vectors $\Pi_{h, z}$ and $\Pi_{e, z}$ take the following forms

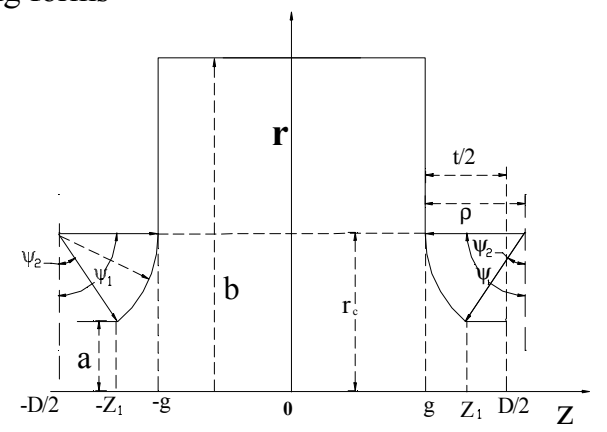

Figure 1 Cross-section of disk-loaded waveguide. 


$$
\begin{aligned}
& \prod_{e, z}^{i n}=-\sum_{n=-\infty}^{+\infty} \frac{A_{n}}{\chi_{n}^{2}} \frac{J_{m}\left(\chi_{n} r\right)}{J_{m}\left(\chi_{n} r_{c}\right)} e^{-j \beta_{n} z} \cos m \theta, \\
& \prod_{h, z}^{i n}=-\frac{1}{Z_{0}} \sum_{n=-\infty}^{+\infty} \frac{B_{n}}{\chi_{n}^{2}} \frac{J_{m}\left(\chi_{n} r\right)}{J_{m}\left(\chi_{n} r_{c}\right)} e^{-j \beta_{n} z} \sin m \theta,
\end{aligned}
$$

where $\chi_{n}{ }^{2}=k^{2}-\beta_{n}{ }^{2}, \beta_{n}=\beta_{0}+2 \pi n / D, \beta_{0}=\phi_{0} / D . J_{m}$ is the first kind Bessel function of order $m$. When $\chi_{n}$ becomes imaginary, the regular Bessel functions can be replaced by modified Bessel functions $I_{m}$ of a real argument. The above Hertz vectors fulfill the Floquet condition that is required by the trial function in eq. (1).

In the outer standing wave region, we have

$$
\begin{aligned}
& \prod_{e, z}^{\text {out }}=-\sum_{s=0}^{+\infty} \frac{C_{s}}{\Gamma_{s}^{2}} \frac{R_{m}\left(\Gamma_{s} r\right)}{R_{m}\left(\Gamma_{s} r_{c}\right)} \cos \alpha_{s}(z+g) \cos m \theta, \\
& \prod_{h, z}^{\text {out }}=j \frac{1}{Z_{0}} \sum_{s=0}^{+\infty} \frac{D_{s}}{\Gamma_{s}^{2}} \frac{S_{m}\left(\Gamma_{s} r\right)}{S_{m}\left(\Gamma_{s} r_{c}\right)} \sin \alpha_{s}(z+g) \sin m \theta,
\end{aligned}
$$

where the transverse propagation constant $\Gamma_{s}^{2}=k^{2}-\alpha_{s}^{2}$ with $\alpha_{s}=\pi \mathrm{s} / 2 g$ and

$$
\begin{aligned}
& R_{m}(\Gamma r)=J_{m}(\Gamma r) Y_{m}(\Gamma b)-J_{m}(\Gamma b) Y_{m}(\Gamma r), \\
& S_{m}(\Gamma r)=J_{m}(\Gamma r) Y_{m}^{\prime}(\Gamma b)-J_{m}^{\prime}(\Gamma b) Y_{m}(\Gamma r) .
\end{aligned}
$$

Here, $Y_{m}$ is the second kind regular Bessel function of order $m$. The regular Bessel functions $J_{m}$ and $Y_{m}$ should be replaced by modified Bessel functions $I_{m}$ and $K_{m}$ of a real argument when $\Gamma_{s}$ is imaginary.

The field components, which are used as the trial functions, can be found from the Hertz vectors (2-5) as follows

$$
\begin{aligned}
\mathbf{E} & =\nabla \times \nabla \times \boldsymbol{\Pi}_{e}-j \omega \mu_{0} \nabla \times \boldsymbol{\Pi}_{h}, \\
\mathbf{H} & =\nabla \times \nabla \times \boldsymbol{\Pi}_{h}+j \omega \varepsilon_{0} \nabla \times \boldsymbol{\Pi}_{e} .
\end{aligned}
$$

Fields given by eqs.(2-3) don't satisfy the metal boundary condition. However, fields given by eqs.(4-5) do. The metal boundary condition on the iris can be satisfied by making function $J$ in eq.(1) minimum.

We use different trial functions in the outer region and inner regions. The fields should be continuous across the interface between the two subregions. We can obtain the fields matching conditions by equating the two tangential components of the magnetic fields at the interface. The procedure to make function $J$ in eq.(1) minimum consists of substituting the trial functions into the functional and thereby expressing the functional in terms of coefficients which are the unknowns, such as $\mathbf{A}, \mathbf{B}, \mathbf{C}, \mathbf{D}$ in eqs.(2-5). The functional is then differentiated with respect to each coefficient, and the resulting equation is set to zero.

It is true that the periodicity theorem, as embodied in eqs. (2-3), help us to obtain the waves only for the infinitely long structure. If, however, we know the value of $\beta_{0}$ for each wave, and the proper linear combination of the harmonics composing each wave, we can specify completely the excitation in a finite structure. We merely choose the amplitudes of the various waves so as to match boundary conditions at both ends of the structure.

\section{NUMERICAL RESULULT}

The field series should be truncated when we make $J$ minimum. Figure 2 shows the value of $J$ around the resonant frequency for the case of disk-loaded structure with round edge beam hole. All examples used in this paragraph have phase 120 degree and structure geometry $t=5.0013 \mathrm{~mm}, D=3.50088 \mathrm{~mm}, b=40.9 \mathrm{~mm}, a=10.0025$ $\mathrm{mm}, \delta=0.5076 \mathrm{~mm}, \rho=2.6007 \mathrm{~mm}$. The number of terms used in the outer region is 53 (s from 0 to 52) and in the inner region 53 (n from -26 to 26 ). $J$ is normalized by $\int\left(Z_{0} \mathbf{H}\right)\left(Z_{0} \mathbf{H}\right)^{*} d V$. It is shown that $J$ takes minimum value at the resonance frequency $2855.9777 \mathrm{MHz}$, which means a true field. The truncation errors decrease when the number of the field terms increases. The convergence of the frequency and field can be estimated from the calculated accuracy of the resonance frequency $\Delta f / f$ and the error of the eigenvector $\varepsilon$ :

$$
\begin{aligned}
& \frac{\Delta f}{f}=\frac{f_{n}-f_{\infty}}{f_{\infty}} \times 100 \% \\
& \varepsilon=\frac{\int\left(\mathbf{E}_{n}-\mathbf{E}_{n-1}\right)\left(\mathbf{E}_{n}-\mathbf{E}_{n-1}\right)^{*} d V}{\int \mathbf{E}_{n} \mathbf{E}_{n}^{*} d V}
\end{aligned}
$$

where $f_{n}$ is the calculated frequency using $n$ terms, $f_{\infty}$ is the convergence frequency when the number of terms is infinite, and $\mathbf{E}_{\mathbf{n}}$ is the eigenfunction obtained from $n$ terms. Figure 3 shows the resonance frequency, accuracy of frequency and estimated error $\varepsilon$ of the first longitudinal mode for different numbers of terms in the inner region, where $f_{\infty} \approx f_{55}$ is used for the calculation of $\Delta f / f$. Note that the accuracy in figure 3 is not the exact accuracy. Nevertheless it shows that the convergence rate of the eigenvalue is very rapid. Usually, we choose the series term $s$ ranging from 0 to 52 in the outer region and $n$ from -26 to 26 in the inner region, which correspond to the total number of terms 53 in Figure 3, to reach an eigenvalue convergence $\delta k / k$ better than $1 \times 10^{-6}$. The estimated error of the eigenvector also approximately decays exponentially with the number of terms. The error can reach to $10^{-6}$ when $n$ is from -26 to 26 . The CPU time is mainly decided by the number of terms in the inner region. The number of terms in the outer region has little effect. We should choose a suitable number of terms in order to get accurate results within a short calculation time. For the above case, The CPU time needed for LONGTRANSVRS to reach within $0.07 \mathrm{MHz}$ of its convergence is about $96 \mathrm{sec}$ while the CPU time for SUPERFISH to reach the same precision is about 4196 sec using a $500 \mathrm{MHz}$ Pentium-III computer. Figure 4 shows calculated frequency and CPU time used for the SUPERFISH code with different mesh sizes and LONGTRANSVRS code with different numbers of terms number as in Figure 3. 


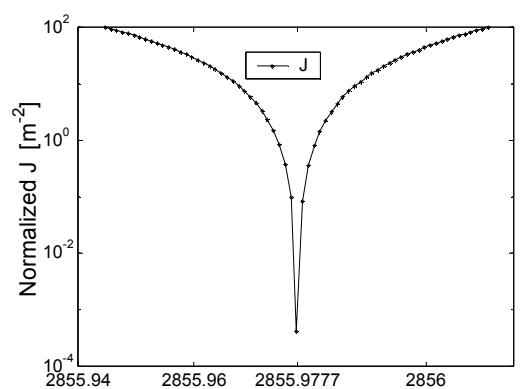

Figure 2 Normalized J vs. frequency

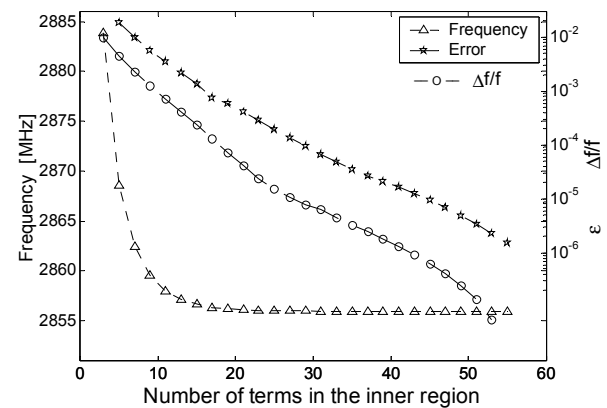

Figure 3 Convergence of frequency and field

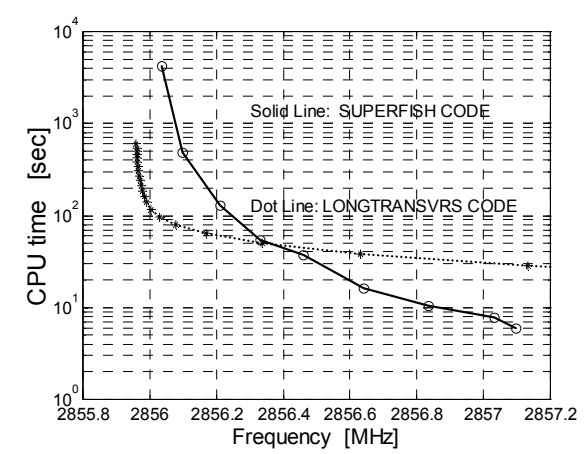

Figure 4 CPU time versus frequencies calculated by the

SUPERFISH code and LONGTRANSVRS code.

LONGTRANSVRS code gives practically the same frequency as TRANSVRS code for a structure with rightangled beam hole edges. For a structure with $t=2 \mathrm{~mm}$, $D=8.7474 \mathrm{~mm}, b=10.779 \mathrm{~mm}, a=4.5 \mathrm{~mm}, \phi_{s}=0$, the first dipole mode frequency is $15.9402820364166 \mathrm{GHz}$ by LONGTRANSVRS, which is the same as that by TRANSVRS within $10^{-3} \mathrm{~Hz}$. Figure 5 shows the convergence of frequency and field for the first dipole mode with phase $120^{\circ}$.

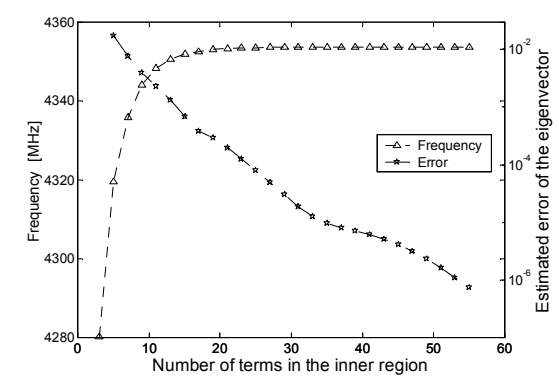

Figure 5 Convergence of frequency and field $(\mathrm{m}=1)$

\section{SUMMARY AND CONCLUSIONS}

Based on the variational method, a code, LONGTRANSVRS was developed which could calculate a disk-loaded structure with rounded edge beam hole shape. It can calculate all modes in disk-loaded structures with high accuracy, which depends on the number of terms used. Our method has good convergence and it's much faster than SUPERFISH code when the accuracy is high. This code provides a powerful tool to design the disc-loaded structure, such as searching for structure geometry ( $a$ or $b$ ) with fixed field modes.

\section{REFERENCES}

[1] M.Takao, et al., "Evaluation of Wake Fields of Disk Loaded Structure for Japan Linear Collider", KEK Report 91-4, 1991.

[2] URSULA VAN RIENEN, "High Order Mode Analysis of Tapered Disc-load Waveguides Using the Mode Matching Technique", Particle accelerators, Vol. 41, pp. 173$201,1993$.

[3] KARL L.F. Bane and Robert L. Gluckstern, "The transverse Wakefield of a Detuned X-band Accelerator Structure", Particle Accelerators, Vol. 42(3-4), pp. 123-169, 1993

[4] S.A. Heifets, S. A. Kheifets, "Longitudinal Electromagnetic Fields in an Aperiodic Structure", SLAC-PUB-5907, 1992.

[5] E. Keil, Nucl. Instrm. Methods, 100, 419, 1972.

[6] K. L. F. Bane and B. Zotter, Proceedings of the XI-th International Conference on High Energy Accelerator, Geneva, 1980.

[7] T. Weiland, Nucl. Instrm. Methods, 216, 329, 1983.

[8] The MAFIA Collaboration, MAFIA.

[9] Z. Li, et al., "Advanced Modeling of RF Cavities and Components for e+ e- Colliders", Proceedings of the First Asian Particle Accelerator Conference, 623, 1998.

[10] K. Halbach and R. F. Holsinger, Particle Accelerator, 7, 213, 1976

[11] M. Yamamoto, "Study of Long-Range Wake Field in Accelerating Structure of Linac", KEK Report 94-9, 1995. 